\title{
Motion Detection Methods in SPECT - A Review
}

\author{
Md. Nahid Hossain and Kamila Afroj Quadir \\ National Institute of Nuclear Medicine \& Allied Sciences, Dhaka, Bangladesh \\ Address For Correspondence: Md. Nahid Hossain, Senior Scientific Officer, National Institute of Nuclear \\ Medicine \& Allied Sciences, BAEC, BSM Medical University Campus, Shahbagh, Dhaka, Bangladesh. \\ Email: nahidhssn@yahoo.com
}

\begin{abstract}
In SPECT, data acquisition occurs over a relatively long time. During the whole acquisition time the patient must lie still, which is very difficult. Therefore, patient movement is frequently reported in clinical applications. These movements cause misalignment of the projection frames and may introduce artifacts. To overcome this, motion detection and correction techniques are applied to improve image quality. In this work, we have reviewed the detection methods for the SPECT motion. Motion detection methods are classified into two categories. These are either external or internal base motion detection. External motion detection are based on hardware using external device which also independently corrects artifacts due to motion. Motion detection data information is separate from the actual image data. Internal motion detection occurs from software or algorithm base using the measured data itself to detect motion. Both external and internal base methods are effective for motion detection in SPECT.
\end{abstract}

Key words: Motion detection, Motion tracking system, Image reprojection.

\section{INTRODUCTION}

Single Photon Emission Computed Tomography (SPECT) is one of the major nuclear medicine imaging modality used for diagnostic imaging of different organs of human body assessing physiological function. A short-lived radiopharmaceutical is injected into the patient body and a series of two dimensional (2D) images of radionuclide distribution are collected using the rotating gamma camera. These data are then reconstructed for three dimensional (3D) distribution of radioactivity to produce a stack of transverse slices of the organ. Patient movement during the acquisition time is a well-known cause of artifacts in reconstructed SPECT data. The data acquisition occurs over a relatively long time, typically in the range of 5-30 minutes. The movements cause misalignment of the projection frames, which degrades the reconstructed image and may introduce artifacts. These motion artifacts may significantly affect the diagnostic accuracy. Fundamentally, motion correction is the task of obtaining consistent projection data from the acquisition. When motion occurs between discrete acquired projections, consistency is lost and errors will be generated to the reconstructed estimate. Therefore, the motion detection and correction of patients in tomography images is very much essential for accurate diagnosis.

Mainly there are two steps in any method for solving the motion problem in SPECT imaging. The first step is motion detection, which estimates the motion of the study, and the second step is motion correction where information from the detection step is applied to compensate for motion and produce a more consistent set of data. Detection and correction are done separately, though most of the authors express the methodologies of detection and correction together when they present a motion correction approach because in many cases, techniques for detection and correction are interrelated.

A number of methods have been developed for solving the motion problem in SPECT. This paper is attempting to broadly categorized the detection methods for the different types of patient motion. Patient motion can be classified in various categories such as rigid body and non-rigid motion. Respiratory motion with associated movement of other organs and diaphragm, cardiac creep and abdominal motions are all examples of non-rigid motions, which arise during imaging. Other type of motion is expressed as vibration motion such as head motion, which is induced by cardiac, or respiratory motion, coughing, sneezing, pain 
reflexes, tremor, and other movement disorders and slow rotational and translational drifting motion.

\section{MOTION DETECTION METHODS}

Motion detection techniques are classified into two categories. These are either external or internal base motion detection. External motion detection are based on hardware such as optical camera with reflective marker, mechanical tracking arm or any external device used for detection which also an independent measurement of the motion. Motion detection data information is separate from the actual image data. Internal motion correction occurs from software such as data base techniques using the measured data itself to detect motion.

\section{HARDWARE BASE OR EXTERNAL MOTION DETECTION}

A flexible multicamera visual-tracking system was used for detecting motion in cardiac SPECT slices by Joseph E. McNamara et al. (1). The authors investigated the Vicon MX visual-tracking system (VTS), which utilizes near-infrared (NIR) cameras to stereo-image small retroreflective markers on stretchy bands wrapped about the chest and abdomen of patients during cardiac SPECT. The chest markers were used to provide an estimate of the rigid-body $(\mathrm{RB})$ motion of the heart and the abdomen markers were used to provide a signal as part of correction of respiratory motion of the heart. The whole system automatically adapts marker tracking to employ all of the cameras visualizing a marker at any instant. Linna Ma et al. also proposed a method based on visual tracking-system (VTS) to compensate for the patient motion during data acquisition (2). Michael A. Gennert et al. investigated another approach based on optical tracking of the patient using a pair of web cameras to acquire stereo images to detect motion (3). The stereo images were analyzed by a Visual Tracking System (VTS) that detects changes in the stereo images over time to track locations on the patient surface.

R. Beach et al. (4) implemented a Neural Network (NN) approach to decompose monitored patient motion data, gathered during cardiac SPECT imaging using the Polaris stereo-IR real-time motion-tracking system. In this method, successful decomposition of Polaris motion data into rigid body motion (RBM) and respiratory motion (RM) was shown. Patient motion was investigated using the Polaris motion-tracking system in cardiac SPECT imaging by R. Beach et al. (5). This system used passive infrared reflection from small spheres to provide real-time position data. The Polaris system viewed through the SPECT gantry toward the patient's head.

Joyeeta Mitra Mukherjee et al. (6) estimated rigidbody and respiratory motion of the heart from marker-tracking data for SPECT motion correction. The author investigated a technique of obtaining patient motion estimates from retro-reflective markers on stretchy bands wrapped around the chest and abdomen of patients being imaged clinically. Motion signals obtained from the markers consist of at least two components, body motion (BM) and respiratory motion. They developed a method for separating these components from the motiontracking data of each marker using Vicon visual tracking system (VTS), and then report a method for combining the $\mathrm{BM}$ estimated from chest markers to estimate the 6- degree-of-freedom (6-DOF) rigidbody motion (RBM) of the heart. Joyeeta Mitra Mukherjee et al. (7) also investigated the patient motion with a visual tracking system (VTS) for investigating the extent, time, and frequency of respiratory and rigid-body motion in cardiac study. It was also determined whether the motion occurred gradually or in sudden jumps, whether it was sustained, and if it occurred along one or more axes predominantly. The differences in respiratory and body motion (BM) was also studied. During imaging, the patients have stretchy belts wrapped similar to their previous work by using the cameras of the Vicon VTS.

S. S. Gleason et al. (8) have developed an optical landmark-based pose measurement and tracking system to provide 3D animal position data for a SPECT imaging system for laboratory animals. The tracking system employed infrared (IR) markers placed on the animal's head along with strobed IR LEDs to illuminate the reflectors and a stereo 
CMOS camera system acquires images of the markers through a transparent enclosure.

Ahmad Bitarafan et. al. (9) have proposed a new approach for the tracking of respiratory motion and the correction of unwanted respiratory motion by the use of respiratory-cardiac gated-SPECT (RCGSPECT). The authors used a respiratory phantom and a charge-coupled device (CCD) camera for the evaluation of respiratory phase and amplitude for the validation of the respiratory tracking system. One CCD camera was installed on one end of the SPECT table and the respiratory phantom was placed on the other end, opposing the infrared camera. A marker block was placed on the phantom and patient motion was tracked using a reflected infrared signal from the CCD camera.

Philippe P. Bruyant et. al.(10) devised a robust method of tracking patient motion with a visual tracking system (VTS) that provides the estimation of motion. Highly retroreflective spheres were clipped on a garment made of black stretchable fabric. This garment was placed around the chest and the spheres 3-D motion is tracked by stereo techniques. The computer stored the JPEG files generated by optical cameras with synchronization to the SPECT system. Olesen OV et al developed an approach for Motion tracking in medical imaging with a nonvisible structured light tracking method. In the study, the authors measured patient movement through projecting light on patient's face and recording the formed pattern by a camera without attaching any motion detectors on the patient's head.

RR Fulton $(11,12)$ developed a detection method which incorporates a motion tracking system to monitor the position and orientation of the patient's head during acquisition. Correction was achieved by spatially repositioning projections according to measured head movements and reconstructing these projections with a fully three-dimensional (3D) algorithm. This method has been evaluated in SPECT studies of the Hoffman 3D brain phantom performed on a triple head camera with fan beam collimation. Movements were applied to the phantom and recorded by a head tracker during SPECT acquisition. Fully 3D reconstruction was performed using the motion data provided by the tracker.

\section{DATA BASED OR INTERNAL MOTION DETECTION}

\section{Cross-Correlation Techniques}

Many authors used cross-correlation method to detect patient motion in projection space.

Saeed Sarkar et. al. (13) developed a linogram/sinogram cross-correlation method for motion detection and correction in planar and SPECT imaging. In this work, a correlation function based on linograms and sinograms of the projection data was introduced to estimate and compensate for the patient motion. Parabolic fitting of the peak of the correlation function was utilized to improve in the motion-estimation task.

Russell D. Folks et al. (14) has developed an automated patient motion detection method in dynamic renal scintigraphy using cross-correlation method. The motion detection used linear crosscorrelation and applied an algorithm that characterizes the difference between mathematic functions. R.L. Eisner et. al. (15) used the crosscorrelation function to detect patient motion in SPECT imaging. This method used frame-to-frame cross-correlation functions of the summed profiles in the vertical and horizontal directions of the planar images. Pixel shifts between two adjacent frames were determined by performing parabolic fits to the peak of the frame-to-frame correlation functions and the two neighboring points.

Michael K. O'Connor et. al. (16) compared four motion correction techniques in SPECT imaging of the heart using a cardiac phantom. One of the techniques, the cross-correlation method, developed by R.L. Eisner et. al. (15) was used again by the authors and compared with other techniques for motion detection in SPECT imaging.

W.D. Leslie et al. (17) also compared different types of motion correction algorithms for cardiac SPECT. In this work the authors also compared the cross-correlation method which was developed by R.L. Eisner et al. (15) with other techniques for motion detection in SPECT imaging. The authors 
also used a marker and then motion histogram was derived from the marker image data using cross correlation technique.

Pellot-Barakat C (18) investigated the motion detection in triple scan SPECT imaging. This protocol performed the acquisition of three full sets of SPECT data which can be adequately combined in order to reconstitute a motion-free set of projection data. Cross-correlation of the projection sets as a function of angle was used for motion detection. Another process was dual scan protocol which produced motion corrected projections and was used in conjunction with reconstruction method (19).

R. Eisner et. al. (20) analyzed a quantitative study of the Tomographic Thallium-201 myocardial bullseye display correcting for patient motion. The motion quantitation and correction technique was based upon a cross-correlation algorithm which was developed by the authors earlier (R.L. Eisner et. al. 1987) to detect patient motion during a tomographic scan. The authors used the algorithm to analyze only sudden and vertical (axial) motion.

\section{Centre of mass techniques}

William J. Geckle et al. (21) investigated correction of patient and organ movement in exercise thallium - 201 cardiac imaging with SPECT. The authors described a technique for correction of artifact in exercise Tl-201 single photon emission computed tomography (SPECT) images arising from abrupt or gradual translational movement of the heart during acquisition. The procedure involved the tracking of the "center of the heart" in serial projection images using an algorithm which is called "diverging square". Each projection image realigned in the $\mathrm{x}-\mathrm{y}$ plane so that the heart center conformed to the projected position of a fixed point in space.

B. Feng et al. (22) estimated the rigid-body motion from three-dimensional images using a center-ofmass points approach. The authors presented an analytical method for the estimation of rigid-body motion in sets of three-dimensional SPECT and PET slices. This method utilized mathematically defined generalized center-of-mass points in images, requiring no segmentation. It can be applied for compensation of the rigid-body motion in both SPECT and PET, once a series of 3D tomographic images are available. The authors generalized the formula for the center-of-mass to obtain a family of points co-moving with the object's rigid-body motion. From the family of possible points the authors chose the best three points which result in the minimum root-mean-square difference between images as the generalized center-of-mass points for use in estimating motion.

\section{Marker Techniques}

Guido Germano et al. (23) detected the patient motion in dynamic and static myocardial SPECT using $99 \mathrm{~m}-\mathrm{Tc}$ point source marker. The authors developed a method for the detection and correction of translational patient motion in myocardial SPECT studies. The method used a low activity $99 \mathrm{~m}-\mathrm{Tc}$ point source. The source's centroid coordinates were measured or derived for all projection images in a temporal frame. The coordinate curves fitted to predicted distributions and the projection images shifted to realign with the fitted values, with sub-pixel accuracy.

\section{Data-Base or Reconstruction Algorithm Techniques}

Patient motion in SPECT also can be detected and estimated by comparing from the measured projection with the projections generated from reconstruction. Several approaches were developed with this method.

B. F. Hutton et al. (24) developed a 3-D reconstruction/registration algorithm for correction of head motion in emission tomography. A fully three-dimensional (3-D) algorithm was proposed by the authors that estimates the patient motion based on the projection of motion-corrupted data, with incorporation of motion information within subsequent ordered-subset expectationmaximization sub-iterations. $\mathrm{N}$ Jianying $\mathrm{Li}$ et al. (25) proposed an approach with three-dimensional filtered back projection (FBP) reconstruction algorithm in fan-beam SPECT to correct for axial motion (both translation and rotation). The authors 
applied a one dimensional filter kernel to the projections. Hanno Schumacher et al. (26)

developed a algorithm for combined reconstruction and motion correction in SPECT imaging. This unique method was purely based on the measured SPECT data and therefore belongs to the datadriven motion correction algorithm class.

Bing Feng et al (27) introduced a unique method for estimation of 6-degree-of-freedom (6-dof) rigidbody patient motion from projection data by the principal-axes method in iterative reconstruction. The authors developed the method for estimating and compensating rigid-body translations and rotations from scatter and attenuation-compensated projection data in iterative reconstruction when multiple projection angles were acquired at the same time. Both the non-attenuated and attenuated line-integrals were calculated during reconstruction. The data driven motion correction (DDMC) which deals with a full 3D rigid-body motion that occurs between projections is a different approach which was performed by Andre Z. Kyme $(28,29)$. The DDMC approach is applied on comparison of measured and estimated projection data. The reconstruction estimate is updated with the relevant projections using the ordered-subsets expectation maximization (OSEM) algorithm. OSEM allow initial reconstructed estimates to be updated with additional projection information. The procedure repeats until all projection pairs have been incorporated consistently into the reconstruction.

Andre Z. Kyme et al $(28,29)$ introduced the datadriven motion correction method. The authors implemented this method for motion correction in brain SPECT. Data-driven detection and correction of motion was achieved by comparison of acquired data with the forward projections. This enables the brain locations to be estimated and data to be correctly incorporated in a three-dimensional (3-D) reconstruction algorithm. Authors performed the digital and physical Hoffman phantom experiments to explore practical aspects of this approach.

KJ Lee et al. (30) developed an iterative method to correct axial and tangential patient motion occurring during tomographic acquisition. The method uses axial images reconstructed from the uncorrected projection images, which are then forward projected to form a basis for registering the original planar images and, in the process, directly seeking to establish a consistent data set. This method can be applied to all SPECT scans including myocardial and brain SPECT. It was demonstrated that the method is capable of detecting and quantitatively correcting for complex motion in both axial and tangential directions. The phantom experiments result showed excellent resolution and contrast recovery after simulated movement in both the axial and tangential directions and initial results with clinical data sets are encouraging.

\section{DISCUSSIONS}

In this work, we have reviewed the motion detection methods in SPECT from different motion estimation systems proposed by different authors. We have noted that several authors used the motion tracking system to detect the motion using different types of external device. These included the multicamera visual-tracking system, retro-reflective markers, optical camera, neural networking, chargecoupled device (CCD) camera, different types of marker etc. These types of motion detection methods are very effective but the main problem is that for the detection the motion, spare parts or hardware was used and the process was lengthy. However, these are very popular methods for detection motion in SPECT and a lot of authors have done work with external device to solve motion problem. In data base or internal motion detection system, several authors proposed various methods to detect patient motion. These include the cross-correlation method, reprojection technique, frame to frame correlation method with sinogram/linogram techniques and data driven methods. Several authors also proposed some algorithm for estimation of motion and correction. Both external and internal base methods are effective for motion detection.

\section{CONCLUSION}

It is therefore observed from the works reviewed in this paper that a general theme is the use of increasingly sophisticated software and existing 
advanced hardware, and that the field of motion detection and correction in SPECT is very open to future novel ideas (hardware, and especially software) aimed at improving motion detection, characterization and compensation.

\section{ACKNOWLEDGEMENT}

This review work was carried out within the framework of the International Atomic Energy Agency (IAEA) Doctoral Coordinated Research Project (CRP) E2.40.19 on "Advances in Medical Imaging Techniques". The authors would like to acknowledge IAEA for proving the logistic support for this program.

\section{REFERENCES}

1. Joseph E. McNamara, P. Hendrik Pretorius, Karen Johnson, Joyeeta Mitra Mukherjee, and Joyoni Dey. A flexible multicamera visualtracking system for detecting and correcting motion-induced artifacts in cardiac SPECT slices. Am. Assoc. Phys. Med. Med. Phys. 36 (5), May 2009. 1913-192.

2. Linna Ma, Songxiang Gu, Suman Nadella, Philippe P. Bruyant, Michael A. King, Michael A. Gennert. A practical rebinning-based method for patient motion compensation in SPECT imaging. IEEE Proceedings of the Computer Graphics, Imaging and Vision 2005.

3. Michael A. Gennert, Philippe P. Bruyant, Manoj V. Narayanan, Michael A. King. Assessing a System to Detect Patient Motion in SPECT Imaging Using Stereo Optical Cameras. IEEE Proceedings 2003; 1567-1570.

4. Richard D. Beach, Hans Depold, Guido Boening, Philippe P. Bruyant, Bing Feng, Howard C. Gifford, Michael A. Gennert, Suman Nadella, and Michael A. King. An Adaptive Approach to Decomposing Patient-Motion Tracking Data Acquired During Cardiac SPECT Imaging. IEEE Trans. Nucl. Sci. 2007; 54(1): 130-139.

5. R. Beach, H. P. Pretorius, G. Boening, P. P. Bruyant, B. Feng, R. R. Fulton, M. A. Gennert, S. Nadella, and M. A. King, "Feasibility of stereoinfrared tracking to monitor patient motion during cardiac SPECT imaging," IEEE Trans.
Nucl. Sci., vol. 51, no. 5, pp. 2693-2698, Sep. 2004.

6. Joyeeta Mitra Mukherjee, Joseph E. McNamara, Karen L.Johnson, Joyoni Dey and Michael A. King. Estimation of Rigid-Body and Respiratory Motion of the Heart From Marker-Tracking Data for SPECT Motion Correction. IEEE Trans Nucl Sci. 2009 February; 56(1): 147-155.

7. Joyeeta Mitra Mukherjee, Karen L. Johnson, Joseph E. McNamara and Michael A. King. Quantitative Study of Rigid-Body and Respiratory Motion of Patients Undergoing Stress and Rest Cardiac SPECT Imaging; IEEE Trans Nucl Sci. 2010 June 1; 57(3): 1105-1115.

8. S. S. Gleason, J. S. Goddard, M. J. Paulus, J. S. Baba, S. Majewski, M. Smith, T. Tran, A. Weisenberger, B. Welch, R. Wojcik Real-Time, High-Accuracy 3D Tracking of Small Animals for Motion-Corrected SPECT Imaging.

9. Ahmad Bitarafan,Hossein Rajabi, Bernhard Gruy, Feridoon Rustgou, Ali Akbar Sharafi, Hasan Firoozabady, Nahid Yaghoobi, Hadi Malek, Christian Pirich, Werner Langesteger, Mohsen Beheshti; Respiratory Motion Detection and Correction in ECG-Gated SPECT: a New Approach; Korean J Radiol 9(6), December 2008

10. P. Bruyant, M. Gennert, G. Speckert, R. Beach, J. Morgenstem, N. Kumar, S. Nadella, and M. King, "A robust visual tracking system for patient motion detection in spect: Hardware solutions," in IEEE Nucl. Sci. Symp. Conf. Rec., 2004, vol. 5, pp. 3094-3097.

11. Fulton RR, Eberl S, Meikle SR, Hutton BF, Braun M. A practical 3D tomographic method for correcting patient head motion in clinical SPECT. IEEE Trans Nucl Sci 1999; 46(3):667672.

12. Fulton RR, "Correction for Patient Motion in Emission Tomography," Ph.D. dissertation, Univ. Technol., Dept. Appl. Phys., Sydney, New South Wales, 2000.

13. Saeed Sarkar, Mohammad A. Oghabian, Iraj Mohammadi, Alireza Mohammadpour and Arman Rahmim. A Linogram/Sinogram CrossCorrelation Method for Motion Correction in 
Planar and SPECT Imaging. IEEE Transactions on Nuclear Science, Vol. 54, no. 1, February 2007.

14. Russell D. Folks, Daya Manatunga, Ernest V. Garcia and Andrew T. Taylor. Automated Patient Motion Detection and Correction in Dynamic Renal Scintigraphy; J. Nucl. Med. Technol. 2011;39:131-139.

15. Eisner RL, Noever T, Nowak D, Carlson W, Dunn D, Oates J, Cloninger K, Liberman HA and Patterson RE. Use of Cross-Correlation Function to Detect Patient Motion During SPECT Imaging. J Nucl Med. 1987; 28:97-101.

16. Michael K. O'Connor, Kalpana M. Kanal, Mark W. Gebhard and Philip J. Rossman. Comparison of Four Motion Correction Techniques in SPECT Imaging of the Heart: A Cardiac Phantom Study; The Journal of Nuclear Medicine, Vol. 39, No. 12, P 2027-2034, December 1998.

17. W.D. Leslie, Jacqueline O. Dupont, Donna McDonald and Anne E. Peterdy. Comparison of Motion Correction Algorithms for Cardiac SPECT; J Nucl Med. 1997; 38:785-790.

18. Pellot-Barakat C, Ivanovic $M$, Weber DA, Herment A, Shelton DK. Motion detection in triple scan SPECT imaging. IEEE Trans $\mathrm{Nucl}$ Sci 1998; 45(4):2238-2244.

19. Passalaqua AM, Narayanaswamy R. Patient motion correction of SPECT images: dual scan approach. IEEE Proc NSSS'94, Norfolk, VA 1995; 3:1270-1274.

20. Robert Eisner, André Churchwell, Till Noever, Dave Nowak, Karen Cloninger, Daniel Dunn, Wilma Carlson, Joel Oates, Janie Jones, Douglas Morris, Henry Liberman and Randolph Patterson. Quantitative Analysis of the Tomographic Thallium-201 Myocardial Bullseye Display: Critical Role of Correcting for Patient Motion. J Nucl Med. 1988;29: 91-97.

21. William J. Geckle, Terry L. Frank, Jonathan M. Links and Lewis C. Becker. Correction for Patient and Organ Movement in SPECT: Application to Exercise Thallium-201 Cardiac Imaging. J Nucl Med. 1988; 29: 441-450.
22. B. Feng, P. P. Bruyant, P. H. Pretorius, R. D. Beach, H. C. Gifford, J. Dey, and M. A. King. Estimation of the Rigid-Body Motion from Three-Dimensional Images Using a Generalized Center-of-Mass Points Approach; IEEE Trans Nucl Sci. 2006 October ; 53(5): 2712-2718.

23. Guido Germano, Terrance Chua, Paul B. Kavanagh, Hosen Kiat and Daniel S. Berman; Detection and Correction of Patient Motion in Dynamic and Static Myocardial SPECT Using a Multi-Detector Camera; Guido Germano, Terrance Chua, Paul B. Kavanagh, Hosen Kiat and Daniel S. Berman; J Nucl Med. 1993;34:1349-1355.

24. B. F. Hutton, A. Z. Kyme, Y. H. Lau, D. W. Skerrett, and R. R. Fulton. Hybrid 3-D Reconstruction/Registration Algorithm for Correction of Head Motion in Emission Tomography; IEEE Transactions on Nuclear Science, Vol. 49, No. 1, P188-194; February 2002.

25. Jianying Li, Ronald J Jaszczak and R Edward Coleman. A filtered backprojection algorithm for axial head motion correction in fan-beam SPECT. Phys. Med. Biol. 40 (1995):2053-2063.

26. Hanno Schumacher, Jan Modersitzki, and Bernd Fischer; Combined Reconstruction and Motion Correction in SPECT Imaging; IEEE Transactions on Nuclear Science, Vol. 56, No. 1, P73-80; February 2009.

27. Bing Feng and Michael A. King; Estimation of 6-Degree-of-Freedom (6-DOF) Rigid-Body Patient Motion From Projection Data by the Principal-Axes Method in Iterative Reconstruction; IEEE Transactions On Nuclear Science, Vol. 60, No. 3, June 2013.

28. Kyme AZ, Hutton BF, Hatton RL, Skerrett DW, Barnden LR. Practical aspects of a data-driven motion correction approach for brain SPECT. IEEE Trans Med Imag 2003; 22(6):722-729.

29. Kyme AZ, Data driven motion correction in Single Photon Emission Computed Tomography of the Brain M.Sc. thesis, Medical Radiation Physics, University of Wollongong, 2004.

30. Lee KJ, Barber DC. Use of forward projection to correct patient motion during SPECT imaging. Phys Med Biol 1998; 43:171-187. 